\title{
Transplantation of marrow stromal cells to rescue retinal cells in SI-induced retinal degeneration in rats
}

Yuan Guan ${ }^{1,5}$, Lu Cui ${ }^{1,5}$, Yuanyuan Gong ${ }^{2}$, Caihong $\mathrm{Xia}^{3}$, Naihe Jing ${ }^{3}$, Yalan $\mathrm{Wu}^{1,5}$, Chunliang Li ${ }^{5}$, Hua Jiang ${ }^{5}$, Xiaomin Zhong, Xingwei $\mathrm{Wu}^{2, *}$, Weiye $\mathrm{Li}^{1,4,{ }^{*}}$, Guo-Tong $\mathrm{Xu}^{1,5,{ }^{*}}$

${ }^{1}$ Laboratory of Clinical Visual Sciences, Institute of Health Sciences, Shanghai Institutes for Biological Sciences (SIBS), Chinese Academy of Sciences \& Shanghai JiaoTong University School of Medicine, Shanghai, China; ${ }^{2}$ Dept. of Ophthalmology, Shanghai First People's Hospital, Shanghai JiaoTong University; ${ }^{3}$ Laboratory of Molecular Cell Biology, SIBS, CAS; ${ }^{4}$ Dept. of Ophthalmology, Drexel University College of Medicine, Philadelphia, PA, USA; ${ }^{5}$ Key Laboratory of Stem Cell Biology, SIBS, CAS, Shanghai, China

Background: Retinal degeneration is the leading cause of blindness around the world, especially in the developed countries. There is, currently, no cure for such a major eye disease.

Purpose: To investigate the preventive and therapeutic effects of the subretinal transplantation of rat Marrow Stromal derived Cells into rat retinal degeneration model induced by sodium iodate (SI). Methods: Retinal degeneration was induced by single intraveneous injection of SI $(50 \mathrm{mg} / \mathrm{kg})$. Marrow Stromal Cells were isolated from bone marrow aspirates from the femurs of male adult SD rats. Primary culturd MSCs were induced in retinal determination (RD) medium for in vitro differentiation, specific signaling molecules required for eye field specification were added into the RD medium. MSCs were used for transplantation, as donor cells, in the SI treated rats. The therapeutic effects were determined by direct fundus observation, fluorescein fundus angiography (FFA) and electroretinogram (ERG) until sacrifice up to 8 weeks following the cell transplantation. The transplantation efficiency of donor cells was also examined for their survival, integration, and differentiation into retinal cells after the subretinal transplantation at the same intervals.

Results: Retinal pigment epithelium (RPE) and photoreceptors were in apoptosis process and severe damaged after the treatment of SI. The severity of the damage depends on the dosage of SI and the time of the treatment. After MSCs transplanted, CM-DiI labeling donor cells could be observed scattered in the subretinal and the donor cells adopted retinalike morphologies. In a pilot study, in which the SI treated rats were transplanted MSC derived cells, the function of the retina was improved as indicated by ERG examination after the transplantation.

Conclusions: SI treatment to rat is a stable model for the study of retinal degeneration on the mechanism and drug screening. Our primary data showed that the cell transplantation with MSC might be a new therapeutic procedure for retinal degeneration. MSC can act as the reliable donor cell resource of autotransplantation.

Keywords: retinal degeneration, transplantation, ERG

Cell Research (2008) 18:s152. doi: 10.1038/cr.2008.242; published online 4 August 2008

*These three authors contributed equally to this work.

Correspondence: Guo-Tong Xu

E-mail: gtxu@sibs.ac.cn 